\title{
Behavioral Design and Synthesis of 64 BIT ALU using Xilinx ISE
}

\author{
Rajib Chetia, Kaushik Chandra Deva Sarma, Gaurab Baruah \\ (Asst. Professor, CIT, Kokrajhar) (Asst. Professor, CIT, Kokrajhar) \\ (M.Tech student in ECT, Gauhati University)
}

Abstract: This paper presents the behavioral Design and synthesis of a 64 bit ALU. 64 bit ALU is basically a multiplexer that operates mainly 16 operations as per select line Bit-permutation. Flags are other important indicators used for specific purpose e.g. if Sign Flag is HIGH then the output of ALU must be a negative number. $C L R$ can reset the output of $A L U$.

Keywords: Flags, CLR, 64 Bit ALU, VHDL.

\section{Introduction}

VHDL is used as the specification and synthesis language, but the process of transformation is substantially more automated. Specifically, the process of High-level simulation is maintained by the transformation from the level of description into hardware elements being automated. With the Synthesis route, the process of mapping into particular targeted circuit technologies is automated (e.g. Spartan 6E). The Synthesis process therefore both increases productivity (through automation) and reduces flexibility (by restricting the choice of technology.

\section{Behavioral Modeling}

The Behavioral Style architecture contains concurrent statements with sections of sequential statements that describe the outputs of the circuit at a discrete moment in time given particular inputs. While similar language constructs are often found in Dataflow and Behavioral style architectures, only the latter explicitly exhibit the notions of time and control. This style describes the functions of the circuit at the algorithmic level. The highest level of abstraction is the behavioral level that describes a system in terms of what it does (or how it behaves) rather than in terms of its components and interconnection between them

III. ALU Operations:

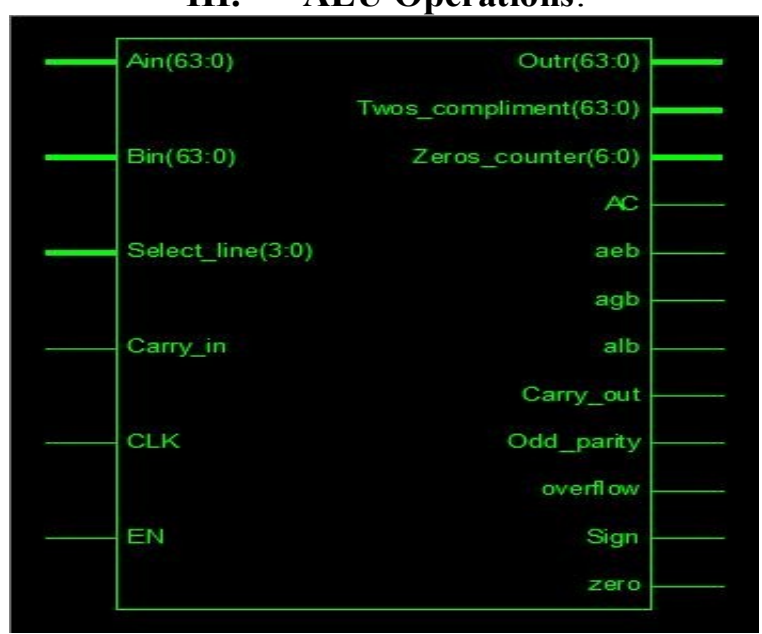

Fig.1.1: Entity 64 Bit ALU

\subsection{Arithmetic Operations}

Carry Look Ahead Adder is a faster adder that overcomes the delay of Ripple Carry Adder. ALU can operate addition; subtraction etc. between Ain and Bin input bus.

\subsection{Logic operations}

All basic logic operations are included e.g. XOR, NAND, NOR, AND etc. 


\subsection{Flags Status}

Flags are too much important in HDL Designing of ALU. All Flags like Sign, Overflow, Carry, Parity, Auxiliary Carry, and Zero. Flags actually allow us to determine the result of an operation more efficiently e.g. Overflow Flag shows occurrence of overflow condition during an operation.

\subsection{Comparator}

ALU compares the inputs Ain and Bin and results whether Ain $>$ Bin, Ain=Bin or Ain $<$ Bin.

\subsection{Others}

Enable, CLR are other parameters that are used to control the operations of ALU. CLR resets the output to logic 0 . If Enable is set to logic 1 the ALU retains the value stored from previous clock cycle.

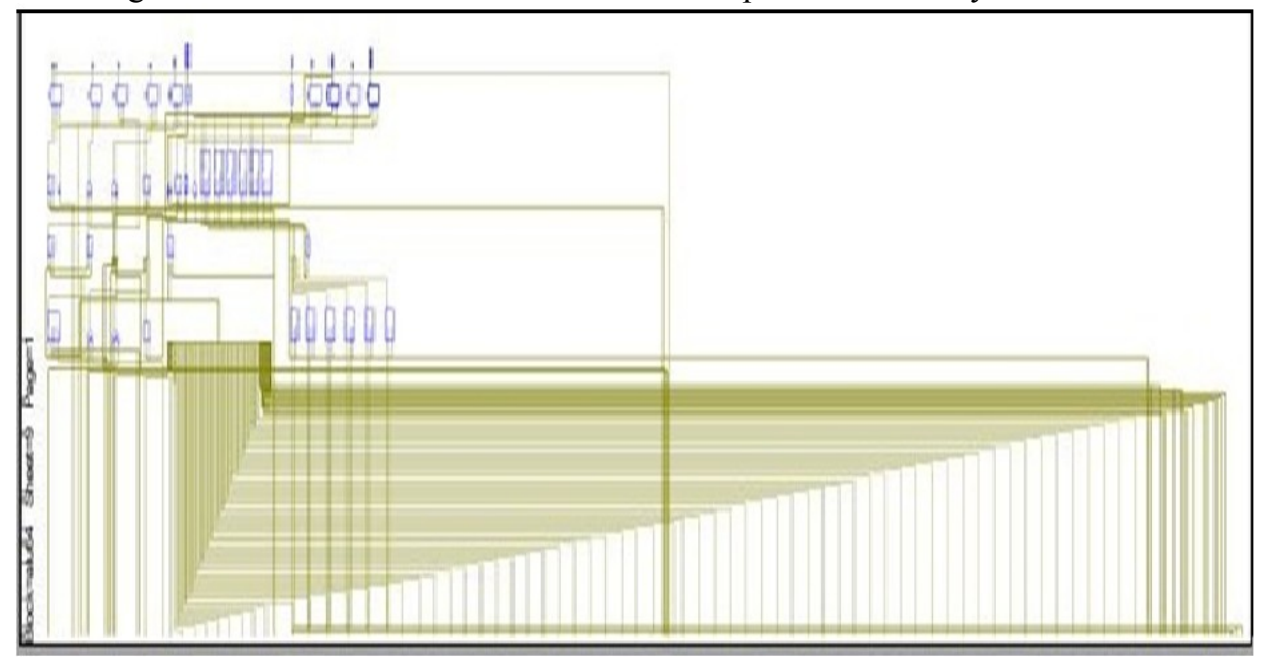

Fig. 1.2: RTL Schematic.

Table 1:

\begin{tabular}{|c|c|c|c|}
\hline $\begin{array}{c}\text { SELECT } \\
\text { LINE }\end{array}$ & OPERATION & SELECT LINE & OPERATION \\
\hline 0000 & Ain AND Bin & 1000 & Ain XNOR Bin \\
\hline 0001 & Ain OR Bin & 1001 & NOT (Ain NAND Bin) \\
\hline 0010 & Ain and (NOT Bin) & 1010 & NOT (Ain NOR Bin) \\
\hline 0011 & NOT Ain & 1011 & Carry Ripple Adder, Carry, Overflow \\
\hline 0100 & NOT Bin & 1100 & Carry Ripple Adder, Carry, Overflow \\
\hline 0101 & Ain NAND Bin & 1101 & 64 Bit Adder \\
\hline 0110 & Ain NOR Bin & 1110 & 64 Bit Subtractor \\
\hline 0111 & Ain XOR Bin & 1111 & Ain NOR (NOT Bin) \\
\hline
\end{tabular}

\section{Xilinx Simulation:}

Simulation of 64 Bit ALU for the Behavioral model has been performed for 1000 nano-seconds (ns). Each Clock cycle has 100 ns rise time and 100 ns fall time. The simulation of 64 Bit ALU(if rising_edge $(C L K)$ and $E N=0)$ generated from Testbench Waveform is given in figure 2.1 and 2.2 below. 


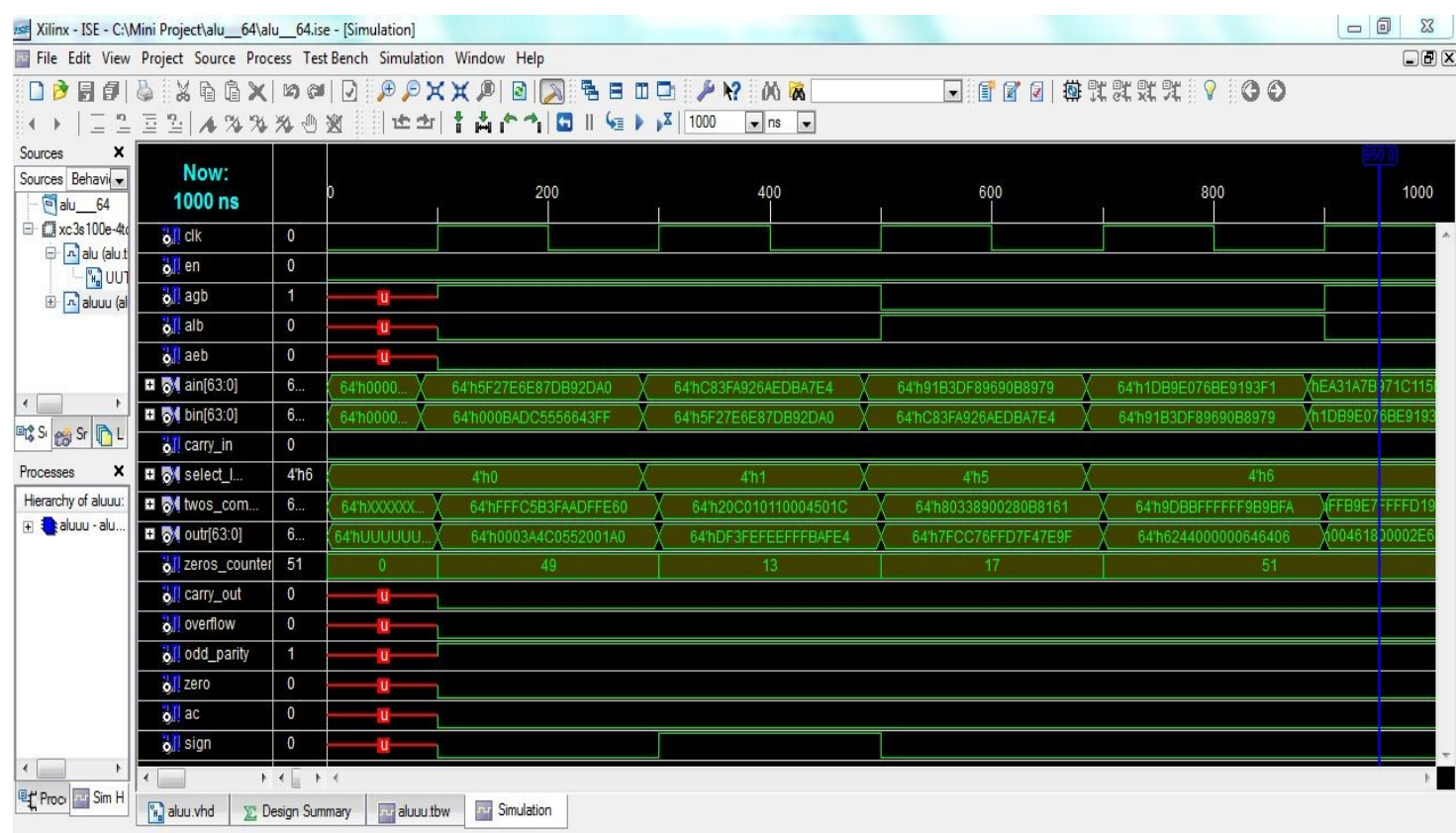

Figure 2.1: Testbench Simulation.

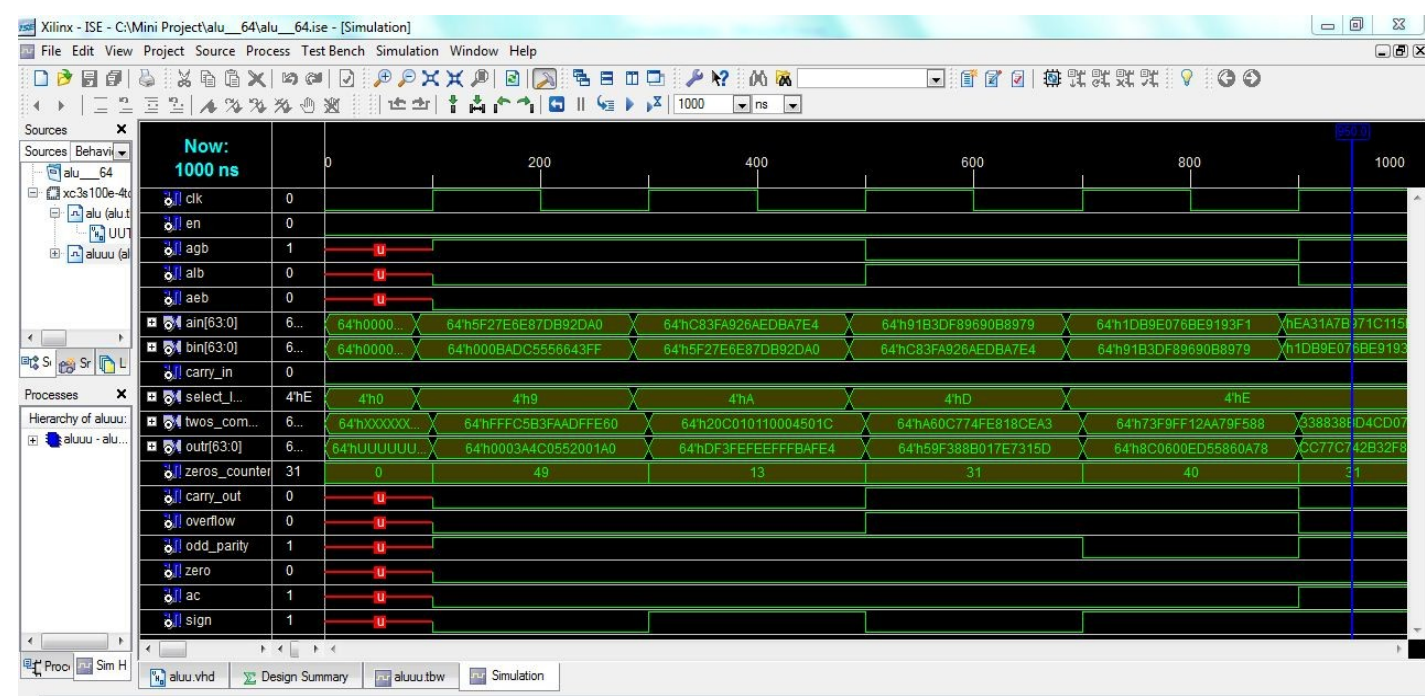

Figure 2.2: Testbench Simulation

V. Synthesis and Implementation Report:

Xilinx-ISE v9.1i has generated the Synthesis and Implementation report for the behavioral model of 64 Bit ALU and are given below: 


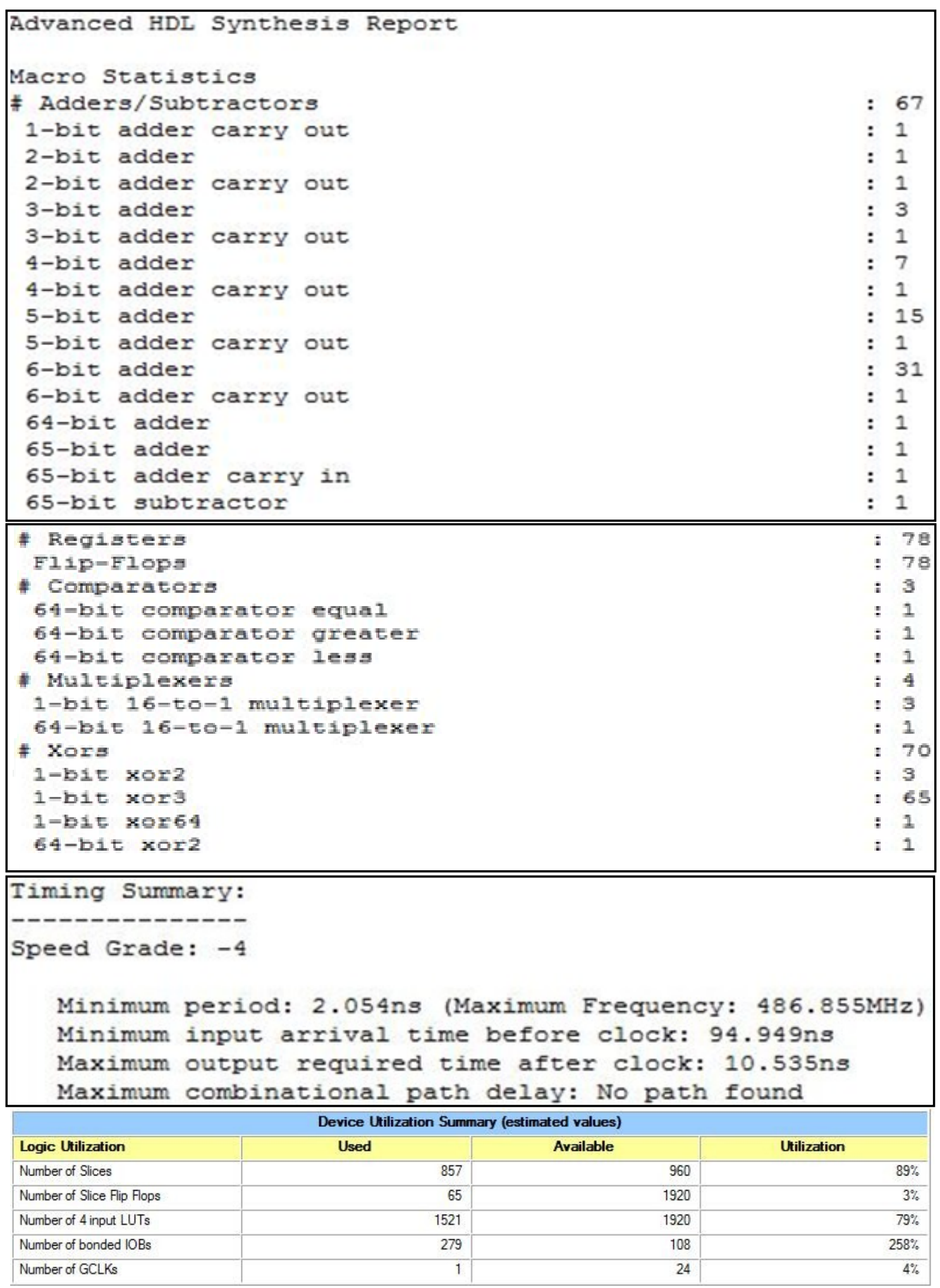

Figure 3: Design Utilization Summary.

\section{Conclusion:}

The 64 bit ALU is designed and synthesized using Xilinx ise v9.1i and targeted to Spartan device. The ALU is a major component of the CPU(Central Processing Unit). It performs arithmetic computation such as Addition, Subtraction, Comparator, Overflow and all basic logical operations (AND, OR, NOT, NOR, XOR, XNOR, NAND). We have verified the results obtained from Xilinx ISE Design Suit v9.1i with the theoretical results for all the operations that were performed and found that they match with the theoretical values. 


\section{References:}

[1]. Suchita Kamble1, Prof .N. N. Mhala "VHDL Implementation of 8-Bit ALU", IOSR Journal of Electronics and Communication Engineering (IOSRJECE) ISSN : 2278-2834 Volume 1, Issue 1 (May-June 2012

[2]. Geetanjali1 and Nishant Tripathi "VHDL Implementation of 32-Bit Arithmetic Logic Unit (Alu)" International Journal of Computer Science and Communication Engineering IJCSCE Special issue on "Emerging Trends in Engineering" ICETIE 2012

[3]. D. Gajski and R. Khun, "Introduction: New VLSI Tools," IEEE Computer, Vol. 16, No. 12, pp. 11-14, Dec. 1983.

[4]. D. L. Perry, "VHDL", Tata Mcgraw Hill Edition, 4th Edition, 2002.

[5]. C. Maxfiled, "The Design Warriors Guide to FPGAs", Elsevier, 2004.

[6]. J. Bhaskar, "VHDL Primer", Pearson Education, 3rd Edition, 2000.

[7]. J. Bhaskar, "VHDL Synthesis Primer", Pearson Education, 1st Edition, 2002. 